\title{
QUANTUM DOT HETEROSTRUCTURES FOR SEMICONDUCTOR DEVICES
}

\author{
1,2,3Nikolay LEDENTSOV \\ ${ }^{1}$ VI Systems GmbH, Berlin, Germany, EU, nikolay.ledentsov@v-i-systems.com \\ 2loffe Institute of the RAS, St. Petersburg, Russian Federation \\ ${ }^{3}$ Academic University, St. Petersburg, Russian Federation
}

https://doi.org/10.37904/nanocon.2019.8446

\begin{abstract}
"Quantum dot (QD)" or, other name, "quantum box", represents an ultimate case of size quantization in solids, where the motion of the charge carriers is restricted in all three dimensions (3D). The critical condition for the formation of truly atom-like density states is a high quality of interfaces surrounding such nano-objects. Defects at interfaces and the related scattering and nonradiative recombination effects drastically reduce or even eliminate advantages of the 3D nanoobjects. Consequently, heteroepitaxial overgrowth became necessary before all the advantages of zero-dimensional electron spectrum in QDs were implemented both in core-shell colloidal QDs and in epitaxially-grown QD heterostructures.
\end{abstract}

Keywords: Quantum dot, semiconductor heterostructure, laser diode, LED, silicon photonics, cryptography, entanglement, nanoelectronics

\section{INTRODUCTION}

Size quantization effects in solids attracted a lot of attention already in 60's of the last century. Such effects were studied first in thin films formed by vacuum deposition [1]. The films formed in this technology had a high density of surface states and defects and, even a lot of bright discoveries were made (such as size quantization of charge carriers, 2D Shubnikov-de Haas oscillations, ...), industrial applications appeared to be hardly possible. In 70's defect-free quantum well (QW) structures were realized [2] and multiple further fundamental discoveries were allowed (fractional Quantum Hall effect, phonon folding and confinement, exciton quantization, ...). Multiple device applications followed as well. The advancement became possible when highly reproducible techniques of epitaxial growth with atomically controlled interfaces such as molecular beam epitaxy (MBE) and metal-organic chemical vapor deposition (MOCVD) were developed and applied.

Like also in the case of thin films, the first quantum-size crystallites ( $\mathrm{CuCl}$ ) were realized in 1981 without surface or interface protection by applying nucleation and growth from oversaturated solid solutions is glass matrices [3]. The interfaces with the surrounding matrix suffered from a poor crystallographic quality. In the same way the surface states dominated the surface and interface properties of colloidal quantum dots (QDs), which were realized in 1983 [4]. Structures with reduced dimensionality were also targeted in traditional epitaxial semiconductor heterostructures. It was quickly recognized that lateral etching of QW structures to form QDs is not an optimal technology due to the high defect density formed at the etched surfaces and interfaces. To overcome the problem and realize defect free structures with reduced dimensionality heteroepitaxial growth was applied. Submonolayer deposition of narrow bandgap material on top of the monolayer-stepped vicinal surfaces resulted in the formation of isolated fractional monolayer domains of narrow gap material [5]. Such domains were then overgrown by the matrix material having a larger bandgap. However, in traditional III-V materials, small effective mass of charge carriers in combination with ultimately small thickness of the insertion, resulted in fairly weak electron and hole confinement by monolayer islands. Furthermore, the interface intermixing effects did not enable clear manifestation of the lateral size quantization effects, and the approach was abandoned for certain period. In the following, the concept was successfully applied to II-VI and III-N materials where large effective masses for charge carriers allow efficient localization of charge carriers already 
at monolayer-thick islands of the narrow bandgap material. In the following research period vertically-correlated stacking of islands formed by sub-monolayer (SML) deposition of narrow bandgap material and separated by layers made of wide bandgap material allowed further bandgap manipulations and engineering of the polarization of luminescence recorded from the surface and the edge of the multilayer SML structures.

As opposite, initially the formation of three-dimensional islands, which may appear during deposition of latticemismatched material on top of a single crystalline substrate was hardly considered as suitable candidate for QD formation or any other high-quality epitaxial growth approach. Such phenomena were broadly studied at the initial phase of MBE and MOCVD technology development. It was concluded that the effect unavoidably results in a high concentration of macroscopic clusters and a generation of high density of dislocations ruining optical properties and potential device performance.

\section{THREE-DIMANSIONAL QUANTUM DOT HETEROSTRUCTURES}

With further progress in heteroepitaxial growth and development of advanced techniques such as submonolayer deposition and heteroepitaxial deposition at reduced temperatures and deposition rates, in combination with applying in-situ techniques for monitoring of epitaxial growth, such as reflection high-energy electron diffraction (RHEED), a much better understanding and control of epitaxial deposition in lattice mismatched systems became possible. Consequently, first defect-free three-dimensional QD heterostructures capable for lasing [6] were finally demonstrated. An explosion of research activity in this field followed. A first clear proof of atom-like electronic spectrum in a real QD was manifested [7]. Characteristic QD absorption features were revealed [7]. Nonequilibrium carrier relaxation processes in QDs became understood [7] and found to be fast enough, as opposite to the initial theoretical concerns. It was further shown that the latticemismatched epitaxial deposition resulted, under certain range of technological parameters, in the formation of size- and shape ordered three-dimensional coherent epitaxial islands having a high degree of order [7]. Selforganized growth of QDs, as formation of order from initially random distributions, was thus demonstrated.

3D colloidal nanocrystallites, with properties initially dominated by surface states, also revealed their broad advantages, when core-shell heteroepitaxial technology [8] was implemented. The surface states of the active QD section became suppressed by an epitaxial wide bandgap protective cover surrounding the narrow bandgap core region. Highly efficient luminescence was demonstrated.

\section{PROOF OF ELECTRONIC QUANTUM DOTS}

Initially properties of InGaAs-GaAs nanostructures remained highly controversial. Until the beginning-middle of 90's many researchers argued that the properties of these structures, such as photoluminescence, are governed by defects and not by the coherent electronic quantum dots. The situation changed in 1993, when the first lasing in self-organized three-dimensional QDs was demonstrated both at low and at room temperature (Figure 1a, according to [6]).

The photoluminescence peak (PL) and the lasing spectrum of QDs at low temperatures were Stokes shifted with respect to the first feature revealed in the PL excitation (PLE) spectrum of the QDs. This effect was in a marked difference to the traditional QW structures where PL and PLE maxima and the lasing spectrum all overlap and the Stokes shift is minor for high quality structures. Such PLE and PL behavior later was understood [7] and further became a fingerprint of a 3D isolated quantum dot. The main reason of such behavior is: resonantly excited QDs at low temperatures into the ground state can emit only at the wavelength matching the excitation wavelength, as no scattering mechanism within the ground state of the QD exists. Such ground state emission is revealed in time-delayed PL studies, otherwise it is masked by the intensity of the excitation light. Only when the excited state of the QD is populated with the existing light, the ground state emission of QDs can be revealed in the PL and PLE spectra. 
An advantage of the research at this stage was a coordinated research in different areas such as photoluminescence, electroluminescence, cathodoluminescence and calorimetric absorption studies on one side and a detailed characterization of structural properties on the other.

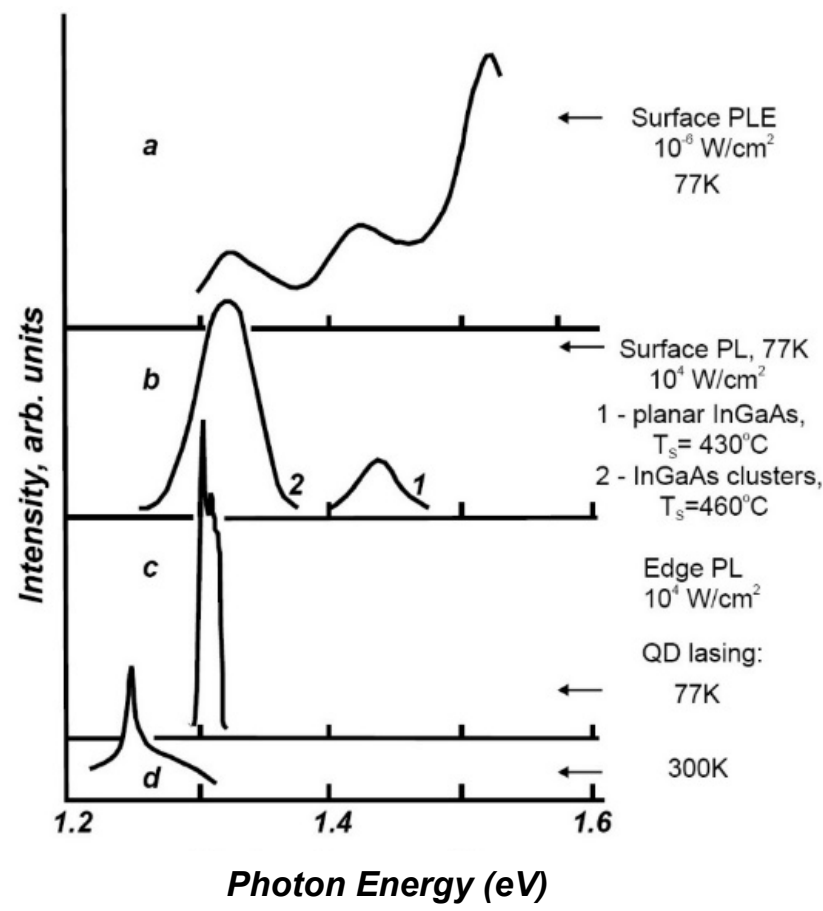

(a)

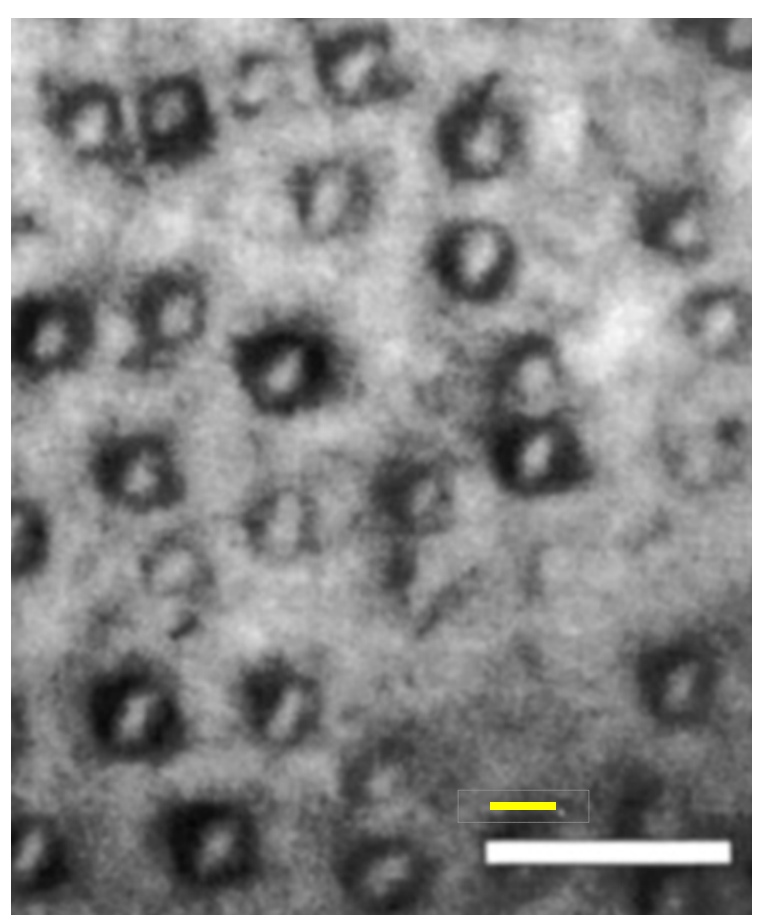

(b)

Figure 1 (a) Optical properties of a structure with InGaAs nanoislands ('quantum clusters' [6]) inserted into a GaAs waveguide layer confined by AIGaAs cladding layers. Photoluminescence (PL) and PL excitation spectra are shown. At high excitation densities, a broad emission peak is revealed in a structure with islands formed by $1 \mathrm{~nm}$ InGaAs deposition at $460^{\circ} \mathrm{C}$. The same nominal InGaAs layer thickness at $430^{\circ} \mathrm{C}$ did not result in the formation of well-resolved 3D islands, and the PL peak was observed at $1.42 \mathrm{eV}$. Photo-pumped lasing in the direction parallel to the surface of the wafer was realized in the structure with the 3D islands at $77 \mathrm{~K}$ and at $300 \mathrm{~K}$ at much longer wavelengths as compared to those for the layer-like distribution of InGaAs

(according to [6], the paper submitted on December 29, 1993) (b) Plan-view weak beam transmission electron microscopy image of 3D quantum dots in the In-Ga-As material system on GaAs substrate.

The QDs have a lateral size of $12 \mathrm{~nm} \pm 2 \mathrm{~nm}$, having axes aligned parallel to [100] and [010]. Along these directions, most of the dots are aligned (according to [7]).

Optical studies revealed unique properties of electronic quantum dots. Structural characterization of QDs formed by InGaAs-GaAs and InAs-GaAs deposition in optimized growth regimes evidenced a narrow size and shape distribution of coherent islands and their relative ordering along certain crystallographic axes (Figure 1b)

It was found further, that even a moderate deviation from the optimal "window" of deposition parameters may indeed result in formation of defects and dislocated clusters. In the following work, however, it was shown that it is possible to eliminate the impact of such defects also in this case by (i) partial overgrowth of the QDs with the wide bandgap material and the following selective evaporation on the not-capped dislocated islands and larger QDs further followed by (ii) high temperature annealing of the capped QDs allowing healing of point defects and their associates and also size and composition manipulations of coherent QDs by partial interface intermixing 
Photoluminescence (PL), PL excitation and lasing spectra confirmed the formation of electronic QDs, even the impact of scattering could not be estimated in the initial studies. Another bright achievement came through a high spectral and spatial resolution cathodoluminescence studies of QDs [7].

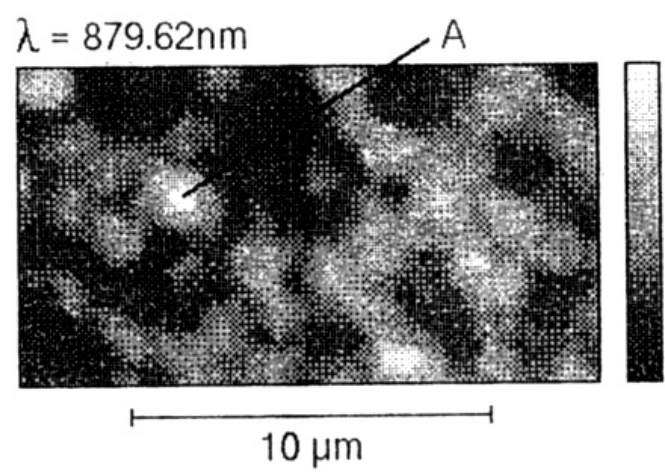

(a)

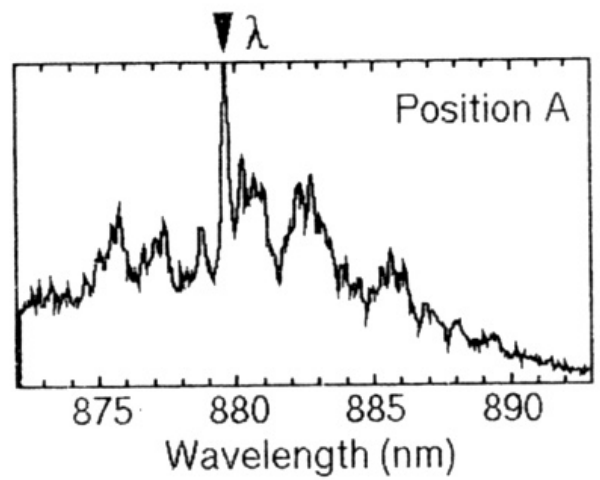

(b)

Figure 2 Intensity distribution map in scanned spot-focus cathodoluminescence (CL) imaging of a sample with InAs QDs taken for particular wavelength within the CL spectrum (a). The spectrum recorded at the maximum intensity position $(A)$ clearly shows a $\delta$-function-like emission line originating at a single nanoisland (b). The size of the bright spot in the intensity map image is defined by the diffusion length of nonequilibrium carriers generated by the electron beam and the electron-hole pair generation volume (according to Reference [7]).

Cathodoluminescence imaging of single QD became a clear proof of formation of truly electronic QD by clear evidencing sharp delta-function like features in the CL spectra (see Figure 2) and the correlation of these sharp features with local excitation areas revealed in CL imaging. Opposite to the case of bound excitons, such sharp features did not disappear with temperature increase. Coherent Lorentzian broadening of the lines at higher temperatures was demonstrated due to phonon-induced interactions. The discovery of sharp emission lines caused by single QDs [7] enabled in the following single photon QD emitters and allowed research and applications in quantum cryptography and entanglement.

The nature of the discrete electron spectrum in QDs stimulated discussions on a fundamental problem of nonequilibrium carrier relaxation in QDs, the issue which was thought to result in severe restrictions for application of QDs in high speed photonic and electron devices.

PL and PLE spectra of InAs nanoisalnds at low temperatures were studied and compared to the absorption spectrum of QDs [7], as presented in Figure 3. It was confirmed that the main inhomogeneously broadened QD PL emission peak is resonant to the feature revealed in the calorimetric absorption spectra (CAS). As opposite no feature at such wavelength was revealed in the PLE spectra. no-transport of nonequilibrium carriers between the QDs and within the forbidden gaps separating the discrete levels of the single QD is posssible. Phonon resonances were revealed in the PLE spectra of QDs having the same energy for the ground state transition- PLE spectrum was inhomogeneously broadened by the shape and size variations of QDs causing a variety of excited state energies for the QDs having the same ground state. Such enhanced broadening of the excited states in QDs was revealed in CAS. PLE features matched LO-phonon energies and their multiples and indicated that the exciton relaxation in QDs occurs faster, when the energy difference between the ground and the excited state in the QD matches the integer of the longitudinal optical (LO) phonon energy. Furthermore, the broadening of the phonon resonances was observed and explained by strain and composition gradients in a single QD, causing effective broadening of the phonon features and by the coexistence of different kinds of phonons for the same QDs (phonons in binary and alloy regions of different composition and within composition gradients, strain gradients, contribution of phonons in wetting layers,...). 
The contribution of interface-folded acoustic phonons further enables fast relaxation processes for excitons and non-equilibrium carriers in practical self-organized InGaAs QDs. Thus there is no need in the precise energy matching to particular bulk phonon energy to enable fast relaxation process even at low temperatures. A high temperatures homogeneous broadening of the QD levels further accelerates the carrier energy relaxation processes.

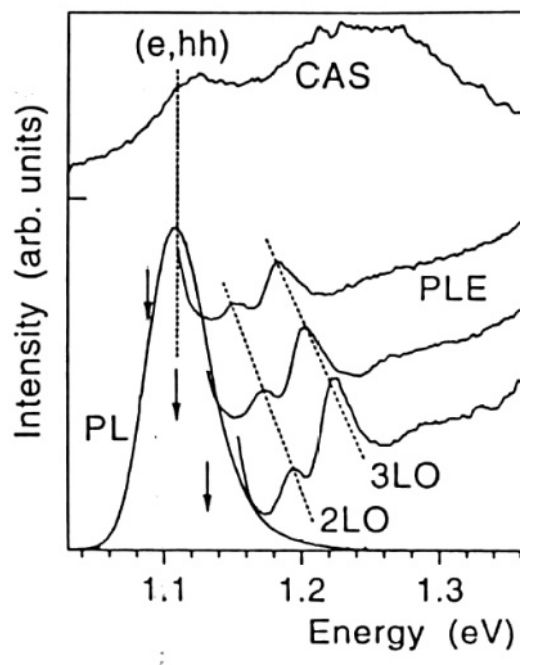

Figure 3 Calorimetric absorption, PL and PLE spectra of 3D InAs QDs in a GaAs matrix (according to Reference [7]).

Heteroepitaxial overgrowth with wide bandgap material was understood to be critical, as it allowed QDs to fully expose their advantages both for fundamental studies and for device applications. Similarly, 3D nanocrystalites revealed their advantages and unique optical properties, when core-shell heteroepitaxial technology [8] was implemented.

\section{DEVICE APPLICATIONS OF QUANTUM DOTS}

All modern QDs for advanced applications are based on the semiconductor heterostructure concept [9]. The major market-relevant applications are:

(i) GaN-based light-emitting diodes (LEDs); where confinement of nonequilibrium carriers at InN-rich nanodomains allows realization of highly efficient LEDs even in case of defect-rich substrates. Control of size and shape of the compositional domains allows adjustment of the color without increase of the nominal $\mathrm{InN}$ composition in the heterostructure avoiding additional defects related to the lattice mismatch.

(ii) QD displays based on core-shell technology enable excellent color gamut, high conversion efficiency and micro LED ( $\mu$-LED) applications including transparent and multi-plane displays. With a better size control of the QDs, different sub-colors in blue, red and green spectral ranges can enable volumetric multi-plane displays via wavelength division multiplexing (WDM) with diffraction grating WDM mirrors.

(iii) QD biomarkers.

Cd-free QDs enable multiple applications in biomarkers allowing multiple applications in medicine and bio-med research

(iv) QDs in laser diodes.

QDs enabled broad $(\sim 100 \mathrm{~nm})$ spectral range multi-wavelength COMB-type lasers at $\sim 1300 \mathrm{~nm}$ and $\sim 1550 \mathrm{~nm}$ wavelengths suitable for multi-channel emission from a single chip. GaAs based high speed uncooled DFB 
lasers are used for applications in passive optical networks (PON) enabling lasers having very low threshold current and transceivers at high energy efficiency. $1300 \mathrm{~nm}$ VCSELs on GaAs substrates (for a review see Ref. [10]) can be applied for optical interconnects in datacenters, and as hybrid light sources for highly integrated silicon photonics. QD VCSELs at $1300 \mathrm{~nm}$ can further replace DFB lasers in GPON and 10 GPON applications in upstream channels. QD heterostructure lasers [10] play a role in the extending of the spectral range from IR [10] to yellow [11] staying within the same GaAs-based material system and technology. Lasers for bio-med applications and displays, including light field displays with wavelength division multiplexing for multiplane images can be targeted.

Among recent advances, one should refer to the realization of a GaAs-based laser emitting at wavelengths shorter than $600 \mathrm{~nm}$ (Figure 4). This indicates a high potential of the technology both in extending the wavelength range of QD lasers and in improving the performance of the existing bright-red devices.

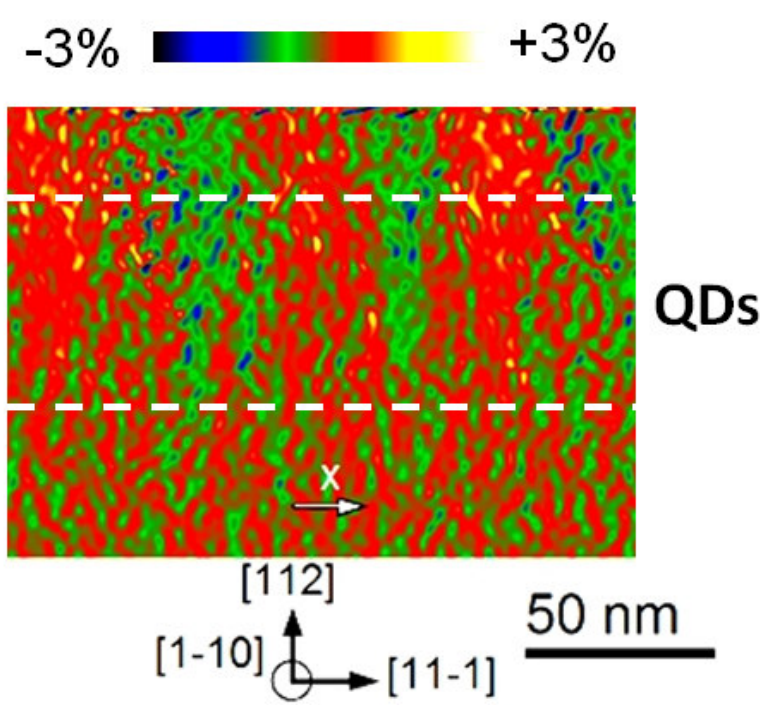

(a)

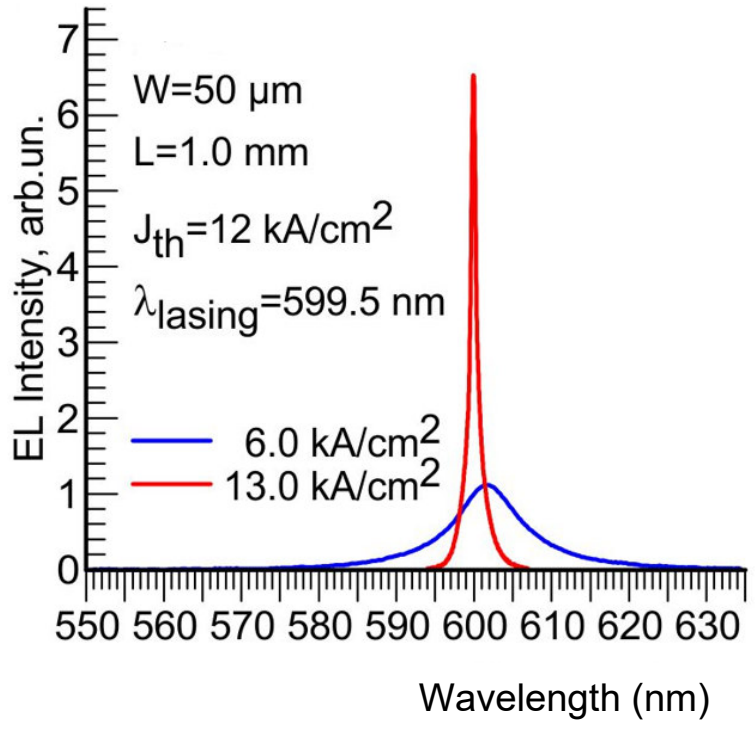

(b)

Figure 4 (a) in-plane $\varepsilon_{x x}$ strain distributions in the gain medium of the QD structure which contains four tensile strained nominally 4-nm thick In ${ }_{0.4} G a 0.6 \mathrm{P}$ layers. Strain was extracted with respect to Ga0.21 Al ${ }_{0.30} \mathrm{In}_{0.49} \mathrm{P}$ lattice with a 4-nm spatial resolution by applying moiré by specimen design technique (MoSD) in the (1-10) cross-section. Note column-like $\varepsilon_{x x}$ strain variation with the column width $\sim 30 \mathrm{~nm}$. Strain field propagate into the region above the $\mathrm{QD}$ area due to the stress-induced phenomena upon overgrowth by into the $\mathrm{Ga}_{0.21} \mathrm{Al}_{0.30} \mathrm{In}_{0.49} \mathrm{P}$ region. Similar columnar arrangement is observed in the (11-1) direction [10,11] (b) Roomtemperature injection lasing in the yellow-orange spectral range. QD based active region of the laser is presented in (a).

For on-board and on-chip optical signaling and, also, for automotive applications, an extreme temperature stability of laser diodes is required. High speed $(25 \mathrm{~Gb} / \mathrm{s}$ and $50 \mathrm{~Gb} / \mathrm{s})$ operation is targeted in a new generation of automotive datacom standards, which address temperatures above $100{ }^{\circ} \mathrm{C}$. QD lasers indeed enable operation at increased temperatures [12] and allow better reliability due to the confinement of nonequilibrium carriers within QDs, preventing nonradiative recombination induced generation and growth of dislocations. Such properties of QDs become of particular critical importance, when microscale light-emitting devices with etched interfaces (for example, microdisc lasers) are implemented.

A TEM image of the QDs applied in the QD VCSEL structure, and an eye diagram at $25 \mathrm{~Gb} / \mathrm{s}$ of the $850 \mathrm{~nm}$ QD VCSEL operating at elevated temperature $\left(170^{\circ} \mathrm{C}\right)$ is shown in Figure 5. 


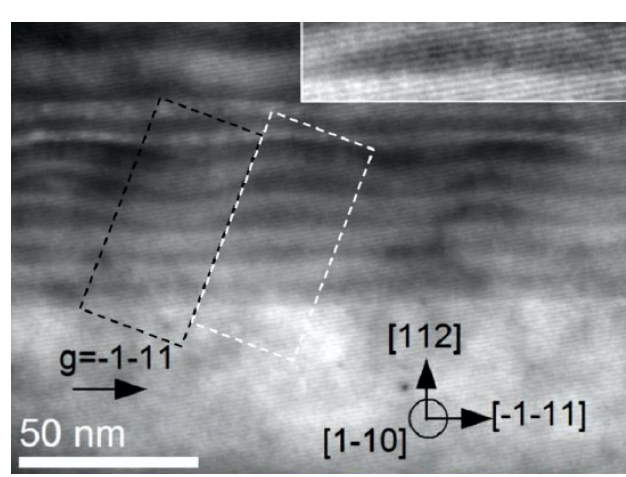

(a)

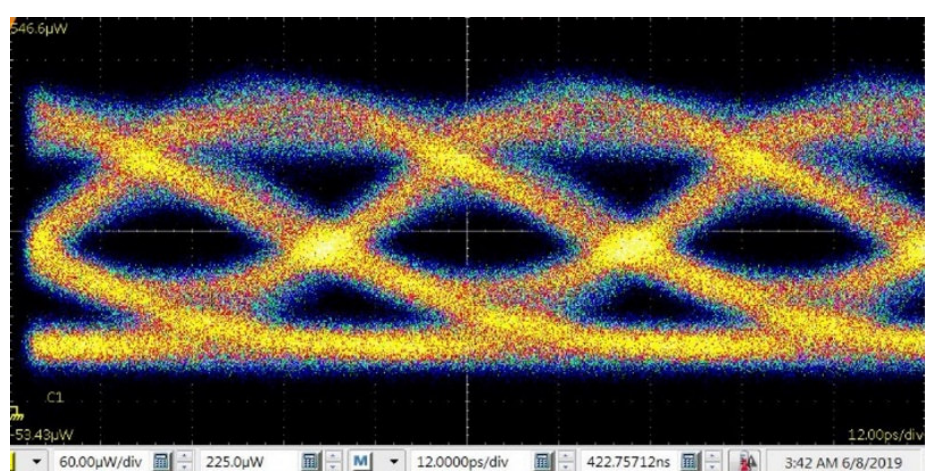

(b)

Figure 5 (a) Bright-field cross-sectional (1-10) Moiré image formed with g=-1-11 of InGaAs QDs in an AIGaAs matrix used as an active region in QD VCSEL; (b) Non-return-to-zero eye diagram of the high temperature [12] $850 \mathrm{~nm}$ QD VCSEL at $25 \mathrm{~Gb} / \mathrm{s}$ measured at $170{ }^{\circ} \mathrm{C}$ chip temperature.

Another application, which requires extreme temperature robustness of the laser, is on-board signaling. IEEE standard for data transmission at $>100 \mathrm{~Gb} / \mathrm{s}$ over the printed circuit board is due beginning of 2021 [13] and the demand for on-board optical interconnects will be further growing.

(v) Quantum communication, sensing, metrology.

A combination of a single QD [7,14], GaAs-based distributed Bragg reflectors (DBR), an oxide confined microresonator and a photonic crystal technology enables a highly directional highly efficient light sources for generation of single or entangled photons suitable for coupling into a single mode fiber.

(vi) QD nanoelectronics and memory devices.

Long storage times for QD heterostructure memory cells are possible, when a proper combination of materials enable high potential barriers for charge carriers preventing their escape [15]. This fact, together with silicon industry reaching characteristic feature size of $\sim 1 \mathrm{~nm}$ provides further opportunities for QD technology. Quantum memory, Coulomb blockade, quantum interference effects and quantum cellular automata enable novel, fast, energy efficient devices and their functional blocks for quantum nanoelectronics of the coming future.

\section{CONCLUSION}

QDs are already broadly applied in multiple key industrial areas and further applications are evolving. Success of QDs in displays and bio-med applications stimulates further acceleration of work in this field. Further applications are in multiwavelength laser diodes, mode-locked lasers for optical synchronization, quantum light sources, telecom range lasers on GaAs, in microlasers. Color of the QDs can be tuned by local on wafer annealing allowing multiwavelength light sources on a single chip. Novel applications of QDs become particularly demanded for the development of novel architecture and concepts for nanoelectronics and on-chip signaling.

\section{REFERENCES}

[1] OGRIN, Yu.V., LUTSKII, V.N., ELINSON, M.I. "Observation of quantum size effects in thin bismuth films" JETP Letters. 3, pp.71-73 (1966).

[2] DINGLE, R.; WIEGMANN, W. and HENRY C. H., "Quantum States of Confined Carriers in Very Thin AlxGa1-xAsGaAs- AlxGa1-xAs Heterostructures" Phys. Rev. Lett. 33, 827-830 (1974). 
[3] EKIMOV, A. I. and ONUSHCHENKO A. A. "Quantum Size effect in three-dimesnional microscopic semiconductor crystals" JETP Lett. 34, 345-349 (1981).

[4] BRUS, L. E. "Electron-electron and electron-hole interactions in small semiconductor crystallites: The size dependence of the lowest excited electronic state". J. Chem. Phys. 80, 4403-4409 (1984).

[5] FUKUI T. and SAITO H. "(AIAs)0.5(GaAs)0.5 fractional-layer superlattices grown on (001) vicinal surfaces by metalorganic chemical vapor deposition Appl. Phys. Lett. 50, 824-827 (1987)

[6] LEDENTSOV, N.N., USTINOV, V.M., EGOROV, A.Yu., ZHUKOV, A.E. MAXIMOV, M.V. TABATADZE, I.G., KOP'EV, P.S. "Optical properties of heterostructures with InGaAs-GaAs quantum clusters" Semiconductors 28 , 832-834 (1994), submitted December 29th 1993.

[7] LEDENTSOV, N.N., GRUNDMANN, M., KIRSTAEDTER, N., CHRISTEN, J., HEITZ, R., BÖHRER, J., HEINRICHSDORFF, F., BIMBERG, D., RUVIMOV, S.S., WERNER, P., RICHTER, U., GÖSELE, U., HEYDENREICH, J., USTINOV, V.M., EGOROV, A.Yu., MAXIMOV, M.V., KOP'EV P.S., and ALFEROV, Zh.I. "Luminescence and structural properties of (In,Ga)As/GaAs quantum dots" Proc. of the 22nd Int. Conf. on the Physics of Semiconductors, Vancouver, Canada, August 15-19,1994, Ed. D.J. Lockwood (World Scientific, Singapore, 1995), p.1855, vol.3

[8] DABBOUSI, B. O., ROdRIGUEZ-VIEJO, J. F., MIKULEC, V., HEINE, J. R., MATTOUSSI, H., OBER, R., JENSEN, K. F., and BAWENDI, M. G. "(CdSe)ZnS core-shell quantum dots: Synthesis and characterization of a size series of highly luminescent nanocrystallites” J. Phys. Chem. B 101(46), 9463-9475 (1997).

[9] ALFEROV, Zh. I. "The history and future of semiconductor heterostructures" Semiconductors 32, 1-14 (1998).

[10] LEDENTSOV N. N "Quantum dot laser" Semicond. Sci. Technol. 26, 014001 (8pp) (2011).

[11] LEDENTSOV, N.N., SHCHUKIN, V.A., SHERNYAKOV, Yu.M., KULAGINA, M.M., PAYUSOV, A.S., GORDEEV, N. Yu., MAXIMOV, M.V., ZHUKOV, A.E., KARACHINSKY, L. Ya., DENNEULIN, T., CHERKASHIN, N. "Room Temperature Yellow InGaAIP Quantum Dot Laser" Solid State Electronics 155, 129-138 (2019)

[12] LEDENTSOV N. Jr, AGUSTIN, M., SHCHUKIN, V.A., KROPP, J.-R., LEDENTSOV, N.N., TURKIEWICZ, J.P., KHAN, Z., CHENG, C.-L., SHI, J.W., CHERKASHIN, N. "Quantum Dot $850 \mathrm{~nm}$ VCSELs with extreme high temperature stability operating at bit rates up to $25 \mathrm{Gbit} / \mathrm{s}$ at $150^{\circ} \mathrm{C}$ " Solid State Electronics 155, 150-158 (2019). LEDENTSOV N. Jr, AGUSTIN, M., KROPP, J.-R., SHCHUKIN, V.A., KALOSHA, V.P., CHI K.L., KHAN, Z.; SHI J.-W., CHERKASHIN, N., LEDENTSOV, N.N. "Quantum dot oxide-confined $850 \mathrm{~nm}$ VCSELs with extreme temperature stability operating at bit rates of $25 \mathrm{Gbit} / \mathrm{s}$ up to $180^{\circ} \mathrm{C}$ " Proc. SPIE Conference OE124 "VerticalCavity Surface-Emitting Lasers XXIV" (2020), accepted abstracts, in print.

[13] IEEE 802.3ck "100 Gb/s per electrical lane" http://www.ieee802.org/3/ck/P802 3ck Objectives 2018mar.pdf

[14] BIMBERG, Dieter "Semiconductor nanostructures for flying q-bits and green photonics" Nanophotonics 7, 12451257 (2018)

[15] MARENTA A., GELLER, M., SCHLIWA, A., FEISE, D., PÖTSCHKE, K. and BIMBERG D. "106years extrapolated hole storage time in GaSb/AIAs quantum dots" Appl. Phys. Lett. 91, 242109 (2007) 